\title{
Constitutive modeling for unsaturated soils considering gas hardening effect
}

\author{
LIU Yan ${ }^{1}$, ZHAO ChengGang $^{1 *}$, CAI GuoQing ${ }^{1} \&$ HUANG Lu ${ }^{1,2}$ \\ ${ }^{1}$ School of Civil Engineering, Beijing Jiaotong University, Beijing 100044, China; \\ ${ }^{2}$ School of Civil Engineering and Architecture, Southwest Petroleum University, Chengdu 610500, China
}

Received March 29, 2010; accepted June 22, 2010

\begin{abstract}
The influence of gases on unsaturated soils is discussed in the paper. First, the selection of stress state variables is discussed. It is shown that gas pressure as well as generalized effective stress and modified suction are required to construct a constitutive model of an unsaturated soil. The deformation mechanisms of solid, liquid and gas phases in soils are then investigated. It is realized that the deformation of gas phase interacts with the deformations of the other two phases in soils. Gas laws are used to describe the gas behavior. Similar to the other two phases in soil, the change of gas volume can be divided into an elastic part and a plastic part, and the latter part is then introduced to the soil hardening equation to reflect the impact of the gas on the soil. Then, a simple elasto-plastic model considering the gas effect for isotropic states is developed. Finally, the model predictions are given and compared with existing experimental data. A good agreement between them is found. Comparisons of the predictions between our model and Wheeler's model are also performed.
\end{abstract}

gas effect, unsaturated soils, elasto-plastic constitutive modeling

Citation: Liu Y, Zhao C G, Cai G Q, et al. Constitutive modeling for unsaturated soils considering gas hardening effect. Chinese Sci Bull, 2011, 56: 1739-1745, doi: 10.1007/s11434-010-4109-0

Almost all the soils on the earth surface are unsaturated. Even in soft soil areas, the soils near the surface are in an unsaturated state. Many engineering problems in China are caused by such unsaturated soils as expansive soils and collapsible loess. More than $60 \%$ of all countries have been, or are being subject to engineering hazards caused by unsaturated soils [1]. Therefore, the fundamental research on the properties and behaviors of unsaturated soils is very important. Usually, unsaturated soils can be treated as a three phase porous material, which consists of solid particles and pores, and the pores are filled with water and gas. Different from metals, the internal structure of unsaturated soils is highly irregular. It is impossible to know the shape and size of every pore. Therefore, it is necessary to adopt a homogeneous macroscopic continuum medium to replace the heterogeneous microscopic porous media. The macro-scopic

*Corresponding author (email: cgzhao@bjtu.edu.cn) continuum medium can then be studied using thermodynamic and mixture theories. The macroscopic continuum medium theory has already been used in unsaturated soils and has been developing rapidly in recent years [2-4]. Zhao et al. [5-7] has done some research in this area and proposed a model framework for unsaturated soils [7]. Based on that framework [7], some simplifications are made to develop an isotropic elasto-plastic model that could consider the gas effect in the present study.

The existence of soil gases (for example, $\mathrm{N}_{2}, \mathrm{O}_{2}$, and $\mathrm{CO}_{2}$ ) makes unsaturated soils different from saturated ones. It increases the compressibility of the fluids, and generates suction at the gas-liquid interface, which makes the properties of unsaturated soils very complicated [8]. In general, soils can be divided into three stages according to the increasing degree of saturation: (1) the continuous gas phase and discontinuous liquid phase stage; (2) the continuous gas phase and continuous liquid phase stage; and (3) the discontinuous 
gas phase and continuous liquid phase stage [9]. Bao et al. [1] pointed out that whether the gas is continuous or not has a great effect on the soil behavior. If gas in the pores is no longer connected to the outward atmosphere, the gas pressure does not remain constant, such as atmospheric pressure. As the occluded gas connects with the solid particles, the variation in gas pressure will affect the soil behavior. The pressure increments of the occluded gas will enhance the soil stiffness. However, this effect is often neglected for simplicity, mainly because of the difficulty of measuring pore-gas pressure in occluded situations and the lack of experimental data. This over-simplification is acceptable for many geotechnical problems, but it cannot be used in marine engineering or other situations that involve large occluded gas pressures and gradients. Loret and Khalili [10] treated the air pressure as an independent variable and developed a three phase model for unsaturated soils. However, their model focused on the mechanical behavior of soils and does not consider the hydraulic hysteresis phenomenon.

This study will first provide the theoretical basis to support the notion that gas pressure should be used as the third stress variable. Then the deformation mechanism of unsaturated soils is discussed. The influence of gases is introduced into the hardening equations, and a simple solid-water-gas coupled model in isotropic state is developed. Finally, the predictions by the model are given and compared with the existing experimental data. The comparisons of the predictions between our model and Wheelers' model without considering gas effect are also given.

\section{Selection of stress state variables}

The selection of stress state variables always plays a central role in developing constitutive models for unsaturated soils. So far, there are mainly three choices: (1) an effective stress like in saturated soils (for example the Bishop stress); (2) two independent stress state variables (for example net stress and matric suction); and (3) work conjugate variables based on the work expression of unsaturated soils [5]. In general, the description of a three phase material needs three independent stress variables: total stress; pore water stress; and pore gas stress [11]. Fredlund and Morgenstern [12] performed a stress equilibrium analysis for an unsaturated soil, and came to the conclusion that the three independent stress variables, i.e. net stress, matric suction and pore gas pressure, were needed to describe the soil behavior under normal conditions. But for simplicity, they did not use the latter stress variable to describe unsaturated soil behaviors, and performed null tests to confirm their viewpoint [12]. From then on, the two independent stress variables theory has been widely used in some unsaturated soil models such as the BBM model [13]. However, Tarantino and Mongiovì [14] have argued that the axis translation technique used in the null test is no longer valid when the air phase is oc- cluded. Consequently, three stress variables instead of two are required. Zhang and Lytton [15] also pointed out that net stress and the matric suction are not a sufficient set of stress state variables (to describe unsaturated soil behavior), because the unsaturated soil status can vary due to the change of air phase volume, even though the net stress and the matric suction are not changed. If the net stress and matric suction are the only two known stress variables, the soil stress state (i.e. the mechanical stress, pore water pressure and pore gas pressure) still cannot be calculated. Therefore these authors suggested that the net stress, matric suction and pore gas pressure should be chosen to describe unsaturated soil behaviors under normal conditions. From the above discussion, it follows that three stress variables instead of two are needed for model completeness.

Based on balance equations for the three phases, Zhao and Zhang [6] derived the work expression for unsaturated soils. They took advantage of mixture theory, combined with the second thermodynamic law, and assumed that there was no interaction among the three phases and that the process was isothermal. Neglecting the diffusion effect, the work equation is the same as the one given by Houlsby [11], but with a stringent theoretical basis and presumptions. The work input can be expressed as [6]:

$$
W=\sigma_{i j}^{*} \mathrm{~d} \varepsilon_{i j}-n s \mathrm{~d} S_{r}+n\left(1-S_{r}\right) u_{a} \mathrm{~d} \rho_{a} / \rho_{a},
$$

where $\varepsilon_{i j}$ is the strain of solid matrix, $S_{r}$ is the degree of saturation, $n$ is the porosity, $u_{a}$ and $u_{w}$ is the pore water pressure and the pore gas pressure, respectively, $s=u_{a}-u_{w}$ is the matric suction, $\rho_{a}$ is the density of gas phase, $\sigma_{i j}^{*}=$ $\left(\sigma_{i j}-u_{a} \delta_{i j}\right)+S_{r}\left(u_{a}-u_{w}\right) \delta_{i j}$ is the effective stress for unsaturated soils.

Eq. (1) indicates that the work input of unsaturated soils consists of three parts: the first part is caused by the deformation of the solid phase, the second part is due to the variation in water content and the third one is induced by the compression of the gas phase. Therefore only two stress variables, namely the net stress and the matric suction are not sufficient, gas pressure is also required as a third stress variable. In this way, three pairs of work conjugate stresses and strains can be used to develop the constitutive model, which are the effective stress, $\sigma_{i j}^{*}$ and the solid strain increment, $\dot{\varepsilon}_{i j}$, the modified suction, $s^{*}=n s$ and the increment of the degree of saturation, $\dot{S}_{r}$, and the gas pressure, $u_{a}$ and the gas volumetric change $\dot{\varepsilon}_{a}=n\left(1-S_{r}\right) \dot{\rho}_{a} / \rho_{a}=$ $-\dot{V}_{a} / V$. Jommi et al. [16-19] among others have used a similar method to construct their model, but they only consider the first two parts of eq. (1), the effect of gas compressibility is neglected.

\section{Constitutive modeling}

Using effective stress, modified suction and gas pressure as independent stress variables, Zhao and Liu [7] developed a 
three phase coupled constitutive framework for unsaturated soils. The model can describe the complexity of unsaturated soil behaviors. In particular, it can consider the influence of gas dissipation on the properties of soils. However, the model is very complex to use in practice; therefore, some simplification is made in this study and a constitutive model for an isotropic state is developed.

\subsection{Deformation mechanism of unsaturated soils}

Wheeler et al. [18] gave a detailed description of the deformation mechanism of the solid and liquid phase for unsaturated soils. They suggested that the elasto-plastic phenomenon of the liquid phase is the hydraulic process of water inflow and outflow to individual voids. For water filled voids, as the suction increases during the drying process, the radius of the curvature of the interfaces between gas and water decreases. This kind of movement of the interfaces is a reversible (elastic) process. If the suction is increased beyond a critical value, where the radius of the interface is just sufficient to bridge the largest entry route to the void, gases will break through into the void. The breakthrough of gases into a void during drying is an irreversible process, because the void will not immediately re-flood with water if the suction is reduced. Similarly, during the wetting process, the flooding of a void with water is treated as an irreversible process. Therefore, air breakthrough into a void, during drying or water flooding of a void during wetting, produces plastic changes in the degree of saturation. A more detailed interpretation of the elastic and plastic mechanism of the liquid phase can be found in Wei and Dewoolkar [20]. Wheeler et al. [18], Sheng et al. [21] and Sun et al. [22] have studied this phenomenon using constitutive models and developed elasto-plastic models for the liquid phase in soils.

Similar to the pore water, the volumetric change of pore gases can also be divided into elastic and plastic parts. Under isothermal conditions, the variation of gas volume is mainly due to the following three reasons: (1) variation of gas mass. Soils undergo mass exchange with the surrounding environment. Gases may be generated by biogenic action, and dissolved in water. All the above factors may change the pore gas volume; (2) external loading and the deformation of the soil skeleton. External loading on the soil will cause soil deformation, which will change the space of pore gas, resulting in a change in gas volume; and (3) the flow of fluids within soil. The inflow and outflow of the pore water directly affects the gas volume in the same pore space. Accordingly, by neglecting the mass exchange, the variation in pore gas volume is mainly due to the deformation of the soil skeleton volume and a change in the degree of saturation. Plastic deformation of soil skeleton may cause irreversible change of pore space, resulting in a plastic change in gas volume. In addition, during the drying-wetting cycles, since the water and gas occupy the same pore space, if a change in the degree of saturation is irre- versible, the corresponding change of gas volume will also be irreversible.

Therefore, irreversible change will occur in all the three phases in unsaturated soils, if the stresses exceed critical values. In an isotropic state, the yield equations of unsaturated soils then can be expressed as

$$
p^{*}=p_{0}^{*}, s^{*}=s_{\alpha}^{*},(\alpha=I, D), u_{a}=u_{a y},
$$

where $p^{*}$ is the mean effective stress. $p_{0}^{*}, s_{a}^{*}$ and $u_{a y}$ are the yield stress. $\alpha=I$ represent the suction increasing yield; $\alpha=D$ represent the suction decreasing yield.

Therefore, similar to Loret and Khalili [10], the strain variables of all the three phases in soils can be divided into elastic and plastic parts:

$$
\mathrm{d} \varepsilon_{v}=\mathrm{d} \varepsilon_{v}^{e}+\mathrm{d} \varepsilon_{v}^{p}, \mathrm{~d} S_{r}=\mathrm{d} S_{r}^{e}+\mathrm{d} S_{r}^{p}, \mathrm{~d} \varepsilon_{a}=\mathrm{d} \varepsilon_{a}^{e}+\mathrm{d} \varepsilon_{a}^{p} .
$$

\subsection{Incremental elasto-plastic relationships}

The elastic deformation is assumed to be dependent on the present stress state, so the elastic relationship can be expressed by the incremental relations. For the soil skeleton:

$$
\mathrm{d} \varepsilon_{v}^{e}=\frac{\kappa}{v} \frac{\mathrm{d} p^{*}}{p^{*}} .
$$

According to Wheeler et al. [18], the elastic relationship of the liquid phase is

$$
\mathrm{d} S_{r}^{e}=-\frac{\kappa_{w} \mathrm{~d} s^{*}}{s^{*}}
$$

Similarly, the elastic relationship of the gas phase can be defined as

$$
\mathrm{d} \varepsilon_{a}^{e}=\frac{\kappa_{a} \mathrm{~d} u_{a}}{u_{a}}
$$

In eqs. (3)-(5), $\kappa, \kappa_{w}, \kappa_{a}$ are elastic constants.

In fact, yielding in one phase will cause deformations of the other two phases, or even cause yielding in the other phases. This coupled effect will be described by the hardening laws in the next section. When yielding in only the solid phase, the plastic volumetric strain is given by

$$
\mathrm{d} \varepsilon_{v}^{p}=\frac{(\lambda-\kappa)}{v} \frac{\mathrm{d} p^{*}}{p^{*}} .
$$

According to Wheeler et al. [18], when yielding in only the liquid phase, the plastic change of the degree of saturation is given by

$$
\mathrm{d} S_{r}^{p}=-\frac{\left(\lambda_{w}-\kappa_{w}\right) \mathrm{d} s^{*}}{s^{*}} .
$$

Similar to liquid phase, the plastic change of gas volume is given by 


$$
\mathrm{d} \varepsilon_{a}^{p}=\frac{\left(\lambda_{a}-\kappa_{a}\right) \mathrm{d} u_{a y}}{u_{a y}} .
$$

In eqs. (7)-(9), $\lambda, \lambda_{w}, \lambda_{a}$ are material parameters. The gas parameter $\lambda_{a}$ can be deduced from the gas laws. Traditionally, the volumetric change in the gases is mainly due to three reasons: compression, dissolution and flow. If the gas is occluded, then volumetric changes will be mainly caused by compression and dissolution. Compressibility can be described by Boyle's law and solubility can be expressed by Henry's law. Therefore, the relationship between the gas pressure and gas volumetric change can be written as [12]:

$$
\mathrm{d} \varepsilon_{a}=n\left(1-S_{r}+H S_{r}\right) \frac{\mathrm{d} u_{a}}{u_{a}}
$$

where $H$ is the Henry index. If solubility is neglected, then $H=0$. Usually, for air $H=0.2$. In fact, $H$ will increase with the gas pressure. In this study, it is assumed to be constant for simplicity.

Comparing eq.(5) with (6), we find that $\lambda_{a}=n\left(1-S_{r}+H S_{r}\right)$. Different from the other two phases, the gas parameter $\lambda_{a}$ is no longer a constant. It will vary with the porosity or the degree of saturation, which confirms that the total gas volumetric change is affected by the solid phase and the liquid phase. Eq. (10) can then be employed in the following sections to develop a constitutive model for unsaturated soils considering the gas effect.

\subsection{Hardening laws}

The hardening laws are used to describe the evolution of the yield surface, and the plastic volumetric strain is mainly used as a hardening parameter to reflect the loading history. Since the solid, liquid and gas phases influence each other, the yield stress of the solid phase is dependent on not only the plastic strain of the soil skeleton, but also the plastic volume change of the liquid and gas. To take these factors into account, the plastic change of $S_{r}$, which reflects the loading history of the liquid phase, and the plastic change of $\varepsilon_{a}$, which reflects the loading history of the gas phase, as well as the plastic volumetric strain, are chosen as hardening parameters. According to Wheeler et al. [18], the hardening equation for the solid phase including the gas effect can be written as

$$
\frac{\mathrm{d} p_{0}^{*}}{p_{0}^{*}}=\frac{v}{(\lambda-\kappa)} \mathrm{d} \varepsilon_{v}^{p}-\frac{k_{s w}}{\left(\lambda_{w}-\kappa_{w}\right)} \mathrm{d} S_{r}^{p}+\eta \frac{k_{s a}}{\left(\lambda_{a}-\kappa_{a}\right)} \mathrm{d} \varepsilon_{a}^{p} .
$$

Soil water retention curves describe the relationship between the degree of saturation and suction and already contain the gas effect. Therefore, the coupled effect between the fluid and gas is not explicitly considered here. The hardening equation for liquid phase is the same as those described by Wheeler et al. [18]:

$$
\frac{\mathrm{d} s_{\alpha}^{*}}{s_{\alpha}^{*}}=\frac{k_{w s} v}{(\lambda-\kappa)} \mathrm{d} \varepsilon_{v}^{p}-\frac{1}{\left(\lambda_{w}-\kappa_{w}\right)} \mathrm{d} S_{r}^{p} .
$$

In eqs. (11) and (12), $k_{s w}, k_{s a}, k_{w s}$ are coupling parameters. $k_{s w}$ controls the magnitude of the shift of solid yield surface caused by liquid phase yielding, while $k_{w s}$ controls the magnitude of the shift of liquid yield surface caused by solid phase yielding [18]. The parameter $k_{s a}$ is introduced to reflect the gas effect, which represents the influence of the gas phase yielding on the solid phase yielding. A detailed method to calibrate these coupled parameters can be found in [23]. $\eta$ in eq. (11) is the gas influence parameter, at $\eta=0$, the degree of saturation is low $\left(S_{r}<0.85\right)$ and the gas effect can be ignored, while at $\eta=1$, the degree of saturation is high $\left(S_{r} \geqslant 0.85\right)$ and the gas is thought to be occluded.

Thus, the gas hardening equation can be derived from eq. (9), where the parameter $\lambda_{a}$ has already contained the influence of solid and liquid:

$$
\frac{\mathrm{d} P_{a}}{P_{a}}=\frac{1}{\left(\lambda_{a}-\kappa_{a}\right)} \mathrm{d} \varepsilon_{a}^{p} .
$$

\subsection{Incremental constitutive relationship}

The increment for the plastic volumetric strain and plastic change of the degree of saturation can be solved explicitly from eqs. (11)-(13):

$$
\begin{aligned}
& \mathrm{d} \varepsilon_{v}^{p}=\frac{(\lambda-\kappa)}{v\left(1-k_{s w} k_{w s}\right)}\left(\frac{\mathrm{d} p^{*}}{p^{*}}-k_{s w} \frac{\mathrm{d} s^{*}}{s^{*}}-\eta k_{s a} \lambda_{a} \frac{\mathrm{d} u_{a}}{u_{a}}\right), \\
& \mathrm{d} S_{r}^{p}=-\frac{\left(\lambda_{w}-\kappa_{w}\right)}{\left(1-k_{s w} k_{w s}\right)}\left(\frac{\mathrm{d} s^{*}}{s^{*}}-k_{w s} \frac{\mathrm{d} p^{*}}{p^{*}}+\eta k_{s a} \lambda_{a} \frac{\mathrm{d} u_{a}}{u_{a}}\right) .
\end{aligned}
$$

Combining eqs. (4) and (14) yields

$$
\begin{gathered}
\mathrm{d} \varepsilon_{v}=\mathrm{d} \varepsilon_{v}^{e}+\mathrm{d} \varepsilon_{v}^{p}=\frac{\kappa}{v} \frac{\mathrm{d} p^{*}}{p^{*}}+\frac{(\lambda-\kappa)}{v\left(1-k_{s w} k_{w s}\right)} \\
\left(\frac{\mathrm{d} p^{*}}{p^{*}}-k_{s w} \frac{\mathrm{d} s^{*}}{s^{*}}-k_{s a} \lambda_{a} \frac{\mathrm{d} u_{a}}{u_{a}}\right),
\end{gathered}
$$

and combining the eqs. (5) and (15) yields

$$
\begin{gathered}
\mathrm{d} S_{r}=\mathrm{d} S_{r}^{e}+\mathrm{d} S_{r}^{p}=-\kappa_{w} \frac{\mathrm{d} s^{*}}{s^{*}}-\frac{\left(\lambda_{w}-\kappa_{w}\right)}{\left(1-k_{s w} k_{w s}\right)} \\
\left(\frac{\mathrm{d} s^{*}}{s^{*}}-k_{w s} \frac{\mathrm{d} p^{*}}{p^{*}}+k_{s a} \lambda_{a} \frac{\mathrm{d} u_{a}}{u_{a}}\right) .
\end{gathered}
$$

The increment stress-strain relationship can be obtained from eqs. (10), (16) and (17),

In practice, we usually know the total stress, pore water pressure and pore gas pressure. Therefore, the stress variables in eq. (18) should be converted using following relationships: 


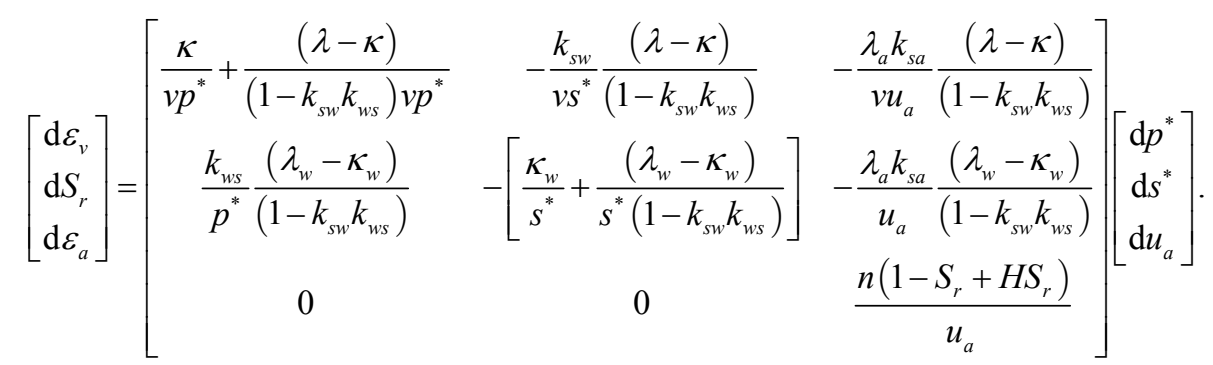

$$
\begin{gathered}
\mathrm{d} p^{*}=\mathrm{d} p-\left(1-S_{r}\right) \mathrm{d} u_{a}-S_{r} \mathrm{~d} u_{w}+s \mathrm{~d} S_{r}, \\
\mathrm{~d} s^{*}=n \mathrm{~d} u_{a}-n \mathrm{~d} u_{w}+s(n-1) \mathrm{d} \varepsilon_{v} .
\end{gathered}
$$

Given the stress variables, the corresponding strain can be calculated by eq. (18), and vice versa. But if the gas phase is occluded, the gas pressure cannot be measured and becomes an unknown variable, resulting in more unknowns than equations. In this case, an additional equation is needed. Considering the deformation compatibility condition, the following relationship can be given,

$$
\begin{aligned}
\mathrm{d} \varepsilon_{a} & =-\frac{\mathrm{d} V_{a}}{V}=-\frac{\left(\mathrm{d} V_{v}-\mathrm{d} V_{w}\right)}{V} \\
& =-\frac{\mathrm{d} V_{v}}{V}+\frac{\mathrm{d}\left(V_{v} S_{r}\right)}{V} \\
& =-\frac{\mathrm{d} V_{v}}{V}+S_{r} \frac{\mathrm{d} V_{v}}{V}+\frac{V_{v}}{V} \mathrm{~d} S_{r} \\
& =\left(1-S_{r}\right) \mathrm{d} \varepsilon_{v}+n \mathrm{~d} S_{r} .
\end{aligned}
$$

In eq. (21), $V$ is the volume of the soil element, $V_{v}$ is the volume of pore space, $V_{a}$ is the gas volume and $V_{w}$ is the water volume. The porosity is given by $n=V_{v} / V$, the degree of saturation is given by $S_{r}=V_{w} / V_{v}$, and the volumetric strain is given by $\mathrm{d} \varepsilon_{v}=-\mathrm{d} V_{v} / V$.

Combining eqs. (18) and (21), a closed equation can be obtained and the stress-strain relationship can be determined. In this model, there are two more parameters than Wheeler's model [18], i.e. the gas parameter $\kappa_{a}$ and the coupled parameter $k_{s a}$.

\section{Results and discussion}

Most existing experiments have been conducted at a low degree of soil saturation $\left(S_{r} \leqslant 0.8\right)$, data for a high degree of saturation are relatively rare because of the difficulty of measuring the gas pressure. Sivakumar and Wheeler [24] have done four series of tests on compacted clays to investigate soil properties. The fourth series with an initial degree of saturation of about 0.8 were chosen to validate our model. Because the gas phase in this series can be assumed to be occluded, and the gas effect is more notable than other series. The initial pressures used were: mean net stress, 50 $\mathrm{kPa}$; pore water pressure, $50 \mathrm{kPa}$; and gas pressures, 150
$\mathrm{kPa}$ or $350 \mathrm{kPa}$. After a wetting stage, isotropic compression was performed on the samples with a constant suction. The model parameters were taken from Wheeler et al. [18] and $\mathrm{Li} \mathrm{[25]} \mathrm{and} \mathrm{are} \mathrm{given} \mathrm{in} \mathrm{Table} \mathrm{1.} \mathrm{The} \mathrm{predicted} \mathrm{results}$ from the model are shown in Figure 1.

Figure 1 gives the test results and the predictions from both our model and Wheelers' model. Both models accurately capture the test data. In the elastic region, the two model curves are overlapped. At a suction of $100 \mathrm{kPa}$, a clear yield point can be found in the figure, while at the suction of $300 \mathrm{kPa}$, no yield point is evident, indicating that suction has a hardening effect and increases the preconsolidation stress. The yield value is greater than the maximum stress applied during consolidation at a higher suction of $300 \mathrm{kPa}$, thus the soil stays in an elastic state. Since the gas effect is considered at the beginning of our analysis, it is evident that in the plastic region our model has a higher yield stress and smaller volumetric changes toward the end. Moreover, the predicted curve for our model is closer to the test points than Wheelers' model, which indicates that the occluded gas does have an effect on the soil. The predicted deformation of gas phase is shown in Figure 2, where a significant yield point can be found, indicating that the change

Table 1 Soil parameters

\begin{tabular}{ccccccccc}
\hline Soil parameters & $\lambda$ & $\kappa$ & $\lambda_{w}$ & $\kappa_{w}$ & $\kappa_{a}$ & $k_{s w}$ & $k_{s a}$ & $k_{w s}$ \\
\hline Figures 1 and 2 & 0.092 & 0.02 & 0.12 & 0.02 & 0.01 & 0.6 & 0.8 & 0.6 \\
Figures 3 and 4 & 0.15 & 0.02 & 0.12 & 0.02 & 0.005 & 0.7 & 0.3 & 0.8 \\
\hline
\end{tabular}

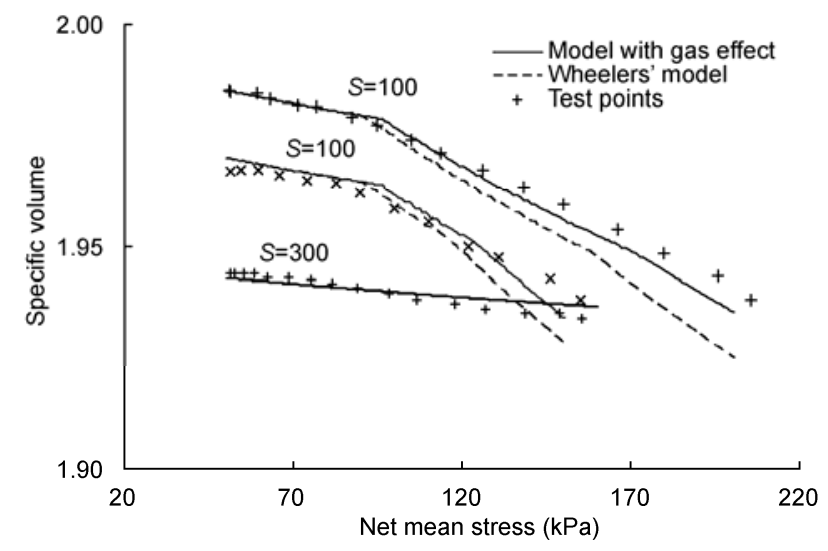

Figure 1 Model simulation of isotropic loading at different constant suctions. 
of gas volume can be divided into an elastic and plastic parts.

Our model is also used to predict the drying-wetting cycle process in Figure 3. The initial degree of saturation is 0.8 . The soil is first dried from point $A$ to $B$, and then wetted from point $B$ to $C$. Then the soil is again dried from point $C$ to $D$ and wetted from point $D$ to $E$. Figure 3 shows that the secondary drying boundary curve $C D$ has drifted to the right compared with the first boundary curve $A B$, and the secondary wetting boundary curve $D E$ has also drifted to the right compared with the first wetting boundary curve $B C$. It is demonstrated that the hydraulic yield value is hardened after a drying wetting cycle.

We then used our model to simulate the water retention behavior, and the results were compared with Wheelers' as shown in Figure 4. The model parameters are given in Table 1. The initial conditions for the model were: $p-u_{a}=10 \mathrm{kPa}$; $v=2.2, S_{r}=0.65, s=200 \mathrm{kPa}$. The net stress was kept unchanged during the modeling process while the suction was initially decreased to $s=10 \mathrm{kPa}$, and then increased back to $s=200 \mathrm{kPa}$. As shown in Figure 4, irreversible compression occurs after drying-wetting cycles, which means the model could accurately reproduce the hydraulic hysteresis phenomenon. In Figure 4(a), the two lines are superposed in the

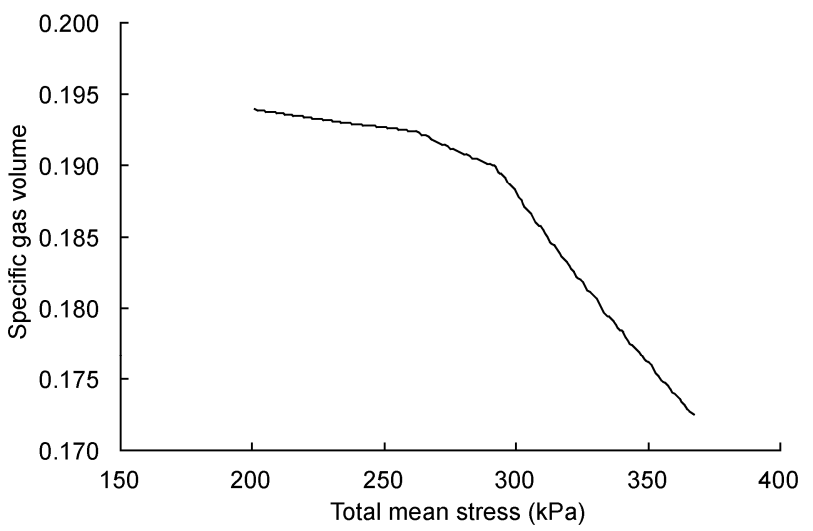

Figure 2 Relationship between gas specific volume and total pressure during compression.

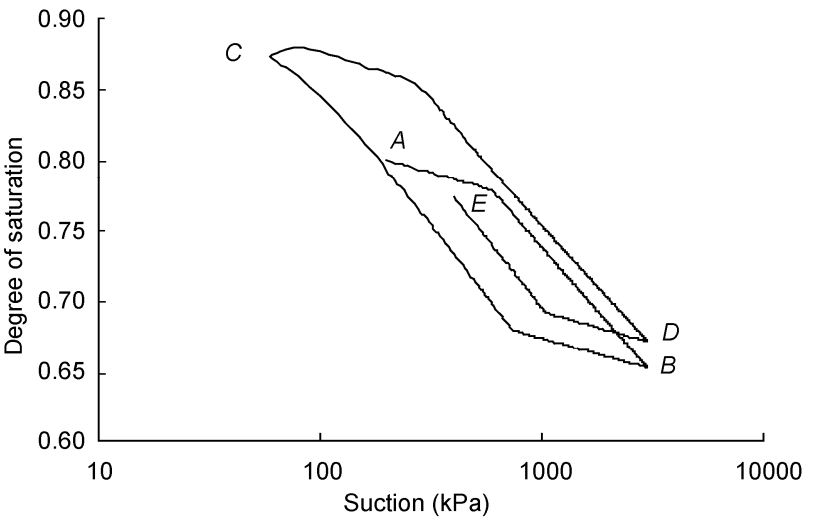

Figure 3 Wetting and drying cycles at constant net mean stress.
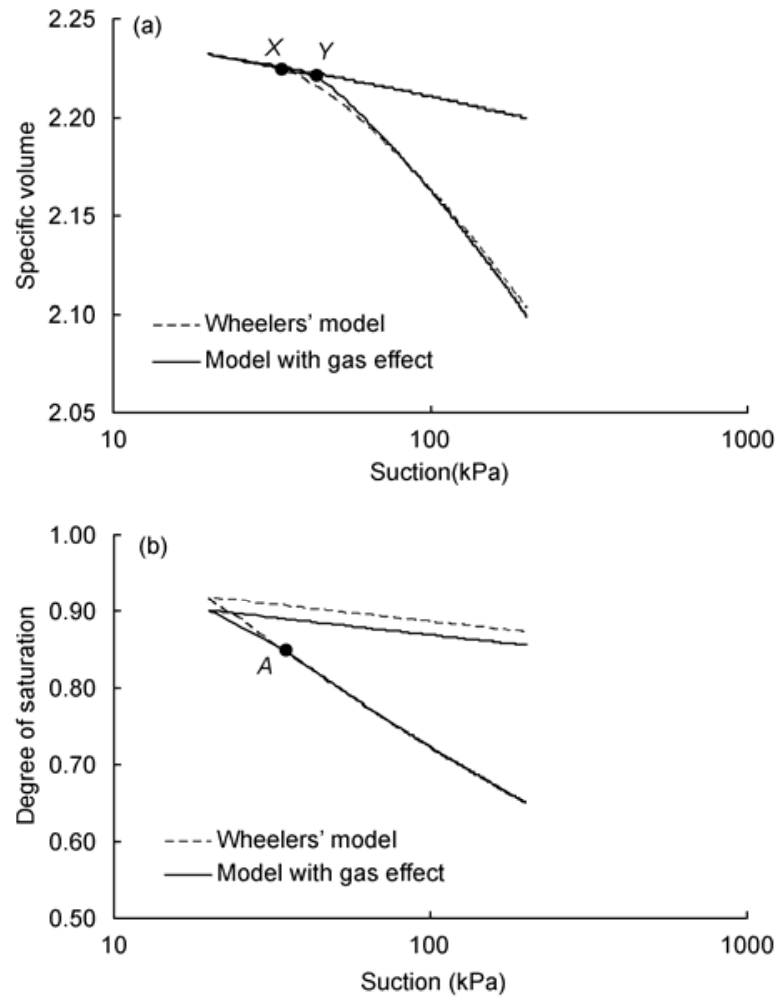

Figure 4 Model simulation of wetting drying cycle at constant net mean stress (a) The relationship between specific volume and suction; (b) the relationship between degree of saturation and suction.

elastic stage, but this is not the case in the plastic stage, because our model has a bigger yield stress (point $Y$ ) than that of Wheelers' model (point $X$ ). No collapse was observed during wetting because our model stays within the LC curve during wetting process. In Figure 4(b), a substantial plastic increase of $S_{r}$ occurs during wetting because of yielding on the $S D$ curve. During the subsequent drying path, only an elastic change of $S_{r}$ occurs, resulting in significant hydraulic hysteresis. In addition, there is no difference between the two models when $S_{r}<0.85$ (before point $A$ ), but when $S_{r}$ $>0.85$, a lower $S_{r}$ is observed in our model than that in Wheelers' model, because the gas effect is considered in our model at this stage. The gas phase is occluded and there are no continuous passages for gas to be expelled, which results in a lower degree of saturation.

\section{Conclusions}

In this study, the selection of stress variables for unsaturated soils is reviewed. It was deduced from the work expression that three stress variables are required in unsaturated soil models to accurately reflect the soil behavior. These are effective stress, modified suction and gas pressure. The corresponding work conjugated strain variable can also be deduced. The deformation mechanisms of the three phases in soils were then discussed. It was shown that gas deformation 
interacts with the deformations of the other two phases. If the degree of saturation is high (usually higher than 0.85), then pore gas will be occluded and the gas pressure cannot be kept constant. Since the occluded gases still connect with the soil skeleton, the variation in gas pressure will affect the soil behavior.

Based on the constitutive framework for unsaturated soil proposed by Zhao and Liu [7], a simplified model in an isotropic state considering gas effect was developed. Using the yield eq. (2), the elastic relations eqs. (4)-(6) and the hardening eqs. (11)-(13), a three phase coupled constitutive eq. (18) for unsaturated soils can be obtained. The experimental data given by Sivakumar and Wheeler [24] were used to validate the model. Comparisons between our model and Wheelers' model are then given, which indicate that the predicted volume change and the variation of the degree of saturation are smaller in our model. It can be concluded that the increase of occluded gas pressure enhances the soil stiffness. The predicted results of the two models agree well with the test data, but our model is slightly more accurate. Our model also shows good capacity in water retention behavior and reproduces the hydraulic hysteresis phenomenon well.

The existence of gases, especially occluded ones, has an effect on the mechanical behavior and deformation properties of soils. Although the gas effect is not significant in general conditions, a complete theory still need to take it into account. In addition, the gas effect will become more evident and cannot be ignored deeper in the soil with increasing gas pressure or in marine sediments with gases are generated by biogenic action. However, there is insufficient data describing the gas effect and its importance to unsaturated soils; therefore, further work is required to do some relevant experiments to validate the existing models and adapt them to more complex conditions.

This work was supported by the National Basic Research Program of China (2010CB732100), the National Natural Science Foundation of China (51078019), and the National Natural Science Foundation of Beijing (8112024).

1 Bao C G, Zhan L T. Relationship between unsaturated soil behavior and engineering problems. Chinese J Geotech Engin, 2006, 28: 129 136

2 Schrefler B A. Mechanics and thermodynamics of saturated/unsaturated porous materials and quantitative solutions. Appl Mech Rev, 2002, 55: 351-388

3 Laloui L, Klubertanz G, Vulliet L. Solid-liquid-air coupling in multiphase porous media. Int J Numer Anal Meth Geomech, 2003, 27: 183-206

$4 \mathrm{Li}$ X S. Thermodynamics-based constitutive framework for unsatu- rated soils. 1: Theory. Géotechnique, 2007, 57: 411-422

5 Zhao C G, Liu Y, Gao F P. Work and energy equations and the principle of generalized effective stress for unsaturated soils. Int J Numer Anal Meth Geomech, 2010, doi: 10.1002/nag.839

6 Zhao C G, Zhang X D. Derivation of the work expression and discussion on the effective principle and the phase separation theorem in unsaturated soils. Sci China Ser E: Technol Sci, 2008, 51: 1530-1541

7 Zhao C G, Liu Y. Continuum porous medium soil mechanics and its application in constitutive relationship of unsaturated soils. Chin J Geotech Engin, 2009, 31: 1324-1335

8 Sun J C, Wang G Q, Sun Q C. Crack spacing of unsaturated soils in the critical state. Chinese Sci Bull, 2009, 54: 2008-2012

9 Wroth C P, Houlsby G T. Soil mechanics: Property characterization and analysis procedures. In: Proc 11th Int. Conf. Soil Mech. Found Eng, San Francisco. 1985. 1-55

10 Loret B, Khalili N. A three-phase model for unsaturated soils. Int J Numer Anal Meth Geomech, 2000, 24: 893-927

11 Houlsby G T. The work input to an unsaturated granular material. Géotechnique, 1997, 47: 193-196

12 Fredlund D G, Morgenstern N R. Soil Mechanics for Unsaturated Soils. Beijing: China Architecture and Building Press, 1997

13 Alonso E E, Gens A, Josa A. A constitutive model for partially saturated soils. Géotechnique, 1990, 40: 405-430

14 Tarantino A, Mongiovì L. Experimental investigations on the stress variables governing unsaturated soil behaviour at medium to high degrees of saturation. In: Tarantino A, Mancuso C, eds. Proceedings of an international workshop on unsaturated soils, Trento, Italy. Balkema, 2000. 1-20

15 Zhang X, Lytton R L. Stress state variables for saturated and unsaturated soils. In: Proceedings of the 4th international conference on unsaturated soils, Carefree, USA. ASCE, 2006. 2380-2391

16 Jommi C. Remarks on the constitutive modelling of unsaturated soils. In: Tarantino A, Mancuso C, eds. Proceedings of an international workshop on unsaturated soils, Trento, Italy. Balkema, 2000. 139-154

17 Vaunat J, Romero E, Jommi C. An elastoplastic hydro-mechanical model for unsaturated soils. In: Tarantino A, Mancuso C, eds. In: Proceedings of an international workshop on unsaturated soils, Trento, Italy. Balkema, 2000. 121-138

18 Wheeler S J, Sharma R S, Buisson M S. Coupling of hydraulic hysteresis and stress-strain behaviour in unsaturated soils. Géotechnique, 2003, 53: 41-54

19 Sheng D, Sloan S W, Gens A. A constitutive model for unsaturated soils: Thermomechanical and computational aspects. Comput Mech, 2004, 33: 453-465

20 Wei C F, Dewoolkar M M. Formulation of capillary hysteresis with internal state variables. Water Res Res, 2006, 42: W07405

21 Sheng D, Sloan S W, Gens A. A constitutive model for unsaturated soils: Thermomechanical and computational aspects. Comput Mech, 2004, 33: 453-465

22 Sun D, Sheng D, Xiang L, et al. Elastoplastic prediction of hydro-mechanical behaviour of unsaturated soils under undrained conditions. Comput Geotech, 2008, 35: 845-852

23 Raveendiraraj A. Coupling of mechanical behaviour and water retention behaviour in unsaturated soils. Dissertation for the Doctoral Degree. Glasgow: University of Glasgow, 2009

24 Sivakumar V, Wheeler S J. Influence of compaction procedure on the mechanical behaviour of an unsaturated compacted clay part 1: Wetting and isotropic compression. Géotechnique, 2000, 50: 359-368

25 Li X S. Thermodynamics-based constitutive framework for unsaturated soils 2: A basic triaxial model. Géotechnique, 2007, 57: 423-435

Open Access This article is distributed under the terms of the Creative Commons Attribution License which permits any use, distribution, and reproduction in any medium, provided the original author(s) and source are credited. 